\title{
Estado del arte de las prácticas pedagógicas de los maestros de las escuelas normales superiores y las facultades de educación
}

\section{State of the art of the pedagogical practices of the superior normal schools and the schools of education}

\author{
Yheny Lorena Pineda-Rodríguez ${ }^{1}$ (1) , Yasaldez Eder Loaiza-Zuluaga²
}

\begin{abstract}
Tipología: artículo de revisión
Para citar este artículo: Pineda-Rodríguez, Y. L. y Loaiza-Zuluaga, Y. E. (2018). Estado del arte de las prácticas pedagógicas de los maestros de las Escuelas Normales Superiores y las Facultades de Educación. Praxis, 14(2), 265-285. Doi: http:// dx.doi.org/10.21676/23897856.2914
\end{abstract}

Recibido en julio 16 de 2018

Aceptado en diciembre 13 de 2018

Publicado en línea en diciembre 16 de 2018

\section{RESUMEN}

El presente artículo de revisión pretende identificar, desde un metaanálisis, los aportes derivados de las investigaciones histórico-educativas relacionadas con las escuelas normales superiores y las facultades de Educación colombianas, como instituciones formadoras de docentes y que conforman el estado del arte de las prácticas pedagógicas de los maestros encargados de formar maestros. En esta investigación doctoral adscrita al grupo Maestros y Contextos de la Universidad de Caldas, se transitó metodológicamente desde el nivel exploratorio, descriptivo y explicativo hasta llegar al análisis documental de los 107 textos que dieron como resultado categorías internas. Los documentos analizados, sistematizados e integrados fueron agrupados así en cinco focos de comprensión común: 1) Formación de maestro: un análisis desde el discurso pedagógico; 2) Prácticas pedagógicas y quehaceres del maestro; 3) Contexto educativo de las escuelas normales; 4) Contexto educativo y procesos de acreditación; y 5) Opciones metodológicas: maestros formadores de maestros. Finalmente, se pudo concluir cómo en las prácticas pedagógicas de quienes forman a los docentes, a pesar de las múltiples miradas desde "el deber ser", aún predomina la distancia entre el discurso y el quehacer pedagógico.

Palabras clave: escuelas normales; formación; quehacer; práctica pedagógica.

\begin{abstract}
The present review article aims to identify, from a meta-analysis perspective, the contributions derived from the historicaleducational research related to Normal Schools and faculties of education that function as teacher training institutions. These contributions constitute the state of the art of the pedagogical practices of teachers, teacher trainers; a doctoral research attached to the group "Masters and Contexts" of the University of Caldas. Methodologically, we went from an exploratory, descriptive and explanatory level to a documentary analysis of the 107 texts that resulted in internal categories. The documents analyzed, systematized and integrated, were grouped into five approaches of common understanding. First, teacher training: an analysis of the pedagogical discourse; second, Pedagogical Practices and teacher s' tasks; third,

\footnotetext{
${ }^{1}$ Mg. Universidad de Caldas, Manizales, Colombia. Correo electrónico: yhelopi@gmail.com ORCID: 0000-0002-6168-4245

${ }^{2}$ Post-Ph. D. Universidad de Caldas y UCM, Manizales, Colombia. Correo electrónico: yasaldez@ucaldas.edu.co ORCID: 0000-0003-4215-2267
} 
the context of normal schools; fourth, educational context and accreditation processes; and fifth, methodological options: teacher educators. Finally, it was possible to conclude that a distance still exists between the discourse and the pedagogical task in the pedagogical practices of the teachers.

Keywords: Normal Schools; Training; Performance; Pedagogical Practice.

\section{INTRODUCCIÓN}

El concepto de práctica pedagógica ha sido reflexionado y estudiado en Colombia desde diferentes disciplinas y por diversos autores, quienes han aportado epistemológica y metodológicamente al respecto y son un referente teórico importante para abordar dicha práctica como objeto de estudio. En ese sentido, el presente artículo trasciende una visión meramente legislativa o normativa y se ubica desde la teoría frente al concepto de práctica pedagógica del maestro que se desempeña en la formación de maestros.

El grupo de "La historia de las prácticas pedagógicas en Colombia" ha desarrollado estudios de carácter histórico, desde la arqueología del saber de Michael Foucault, para mostrar el lugar que hasta la fecha había ocupado la práctica pedagógica entre las prácticas de saber. Fue así como, con base en los resultados y experiencias del proyecto, Martínez (1990, p.1-8) expuso, a partir de la teoría pedagógica, la conceptualización sobre la práctica pedagógica. Esta última, además de ser objeto de investigación, se consideró una noción estratégica, dinámica, cambiante, compleja, indefinida como práctica de saber, según el entorno sociocultural que la rodea, la vida cotidiana de la escuela, las relaciones con la práctica política, las teorías o disciplinas que la apoyan, entre otras. Dicha reflexión sobre la práctica pedagógica contempla tres elementos metodológicos en su funcionamiento interno: la institución, el sujeto y el saber pedagógico.
Conjuntamente, para Zuluaga (1999), la práctica pedagógica es una noción que designa cuatro componentes: en primera instancia, los modelos pedagógicos - tanto teóricos como prácticos- utilizados en los diferentes niveles de enseñanza; segundo, la pluralidad de conceptos o espacios teóricos correlativos a esas funciones, pertenecientes a campos heterogéneos de conocimiento, retomados y aplicados por los lenguajes que le prestan la palabra a la pedagogía; tercero, "las formas de funcionamiento de los discursos en las instituciones educativas donde se realizan prácticas pedagógicas" (Zuluaga, 1999, p.38), cuando se demarca el saber pedagógico en una sociedad específica a partir de la práctica pedagógica, de manera que el saber y la práctica se comprometen en los procesos de institucionalización; y cuarto, "las características u orientaciones de las prácticas sociales adquiridas e impresas por la práctica pedagógica en las instituciones educativas de una sociedad dada, que asignan unas funciones a los sujetos de esa práctica" (De Echeverry, 1979, p.1-9; Zuluaga, 1999, p.17).

Por otra parte, De Tezanos, de la Universidad Pedagógica Nacional (CIUP), define la práctica pedagógica como el resultado de la simbiosis entre la teoría y la práctica. Se refiere así a la asociación entre las estructuras que un maestro aprehendió en su formación, desarrolló y hasta dominó, en cuanto a saberes y principios teóricos e históricos que acompañan a la tradición de enseñar con los diversos fragmentos de las estructuras disciplinarias 
que convergen en el oficio, y la actualización de conocimientos relacionados con la especificidad de la profesión de enseñar y con las demandas y aperturas de la gestión del sistema educativo (2015).

Consecuentemente, la práctica pedagógica, desde una visión sociológica, está constituida, según Díaz (1995, p.333-361), discípulo de Berstein, por la comunicación como evento central, conformada por los procedimientos, las estrategias y las acciones que prescriben la comunicación, el acceso al conocimiento, el ejercicio del pensamiento, de la visión y de las posiciones, las oposiciones-disposiciones y las relaciones sociales de los sujetos en el contexto social del campo pedagógico, en la escuela. En ese sentido, la práctica pedagógica del maestro en la escuela gira en torno a una doble función: un orden instruccional o de conocimiento y un orden regulativo o de relaciones sociales.

Para nosotros, el concepto de práctica pedagógica se asume desde la lógica de las investigaciones lideradas por Loaiza, quien afirma que es el estudio de los discursos, las acciones y los escenarios pedagógicos que privilegian los docentes para su desempeño como formadores de maestros. Esto lleva a comprender cómo "el actuar académico debe reconocer la trascendencia de los saberes que circulan en las fronteras de conocimiento en el aula" y las implicaciones de estos en la formación de los educandos, dado que es precisamente allí en donde se "genera una integración entre docente, estudiante, conocimiento y contexto" (Loaiza, Rodríguez y Vargas, 2012). Este autor concluye que "es necesario que se dé una articulación entre el discurso pedagógico, la acción en el aula y el escenario - ambientes de enseñanza y de aprendizaje - que privilegian los maestros y docentes" (Loaiza y Duque, 2017).

\section{METODOLOGÍA}

Los análisis a continuación presentados dan cuenta, desde un metaanálisis, de los aportes derivados de las investigaciones histórico-educativas relacionadas con las escuelas normales superiores y las facultades de Educación, principalmente del ámbito colombiano, como instituciones formadoras de maestros: las primeras para preescolar y básica primaria, y las segundas para educación básica secundaria, media y universitaria. De este modo, el presente artículo de revisión, resultado de una investigación doctoral adscrita al grupo Maestros y Contextos de la Universidad de Caldas y financiada por Colciencias, (Código 785 de 2017) conforma el estado del arte de lo que se ha investigado sobre las prácticas pedagógicas de los docentes que forman maestros, en el contexto de ocurrencia, para identificar el vacío del conocimiento y desde dónde, con rigurosidad, se va a contribuir a la ciencia con un verdadero aporte.

Las investigaciones en mención necesariamente implican poner al día, aclarar, ordenar y comprender mejor la realidad empírica con los conocimientos previos que se tienen sobre el objeto por estudiar. Por lo tanto, la aproximación en este caso inicia, en su orden, en los niveles exploratorio, descriptivo y explicativo para finalmente llegar al análisis documental y estructurar lo que se denomina estado de la cuestión o estado del arte (Ñaupas, Mejía, Novoa y Villagómez, 2014, p.175, 386).

Armando Zubizarreta (como se citó en Naupas et al., 2014, p.432) afirmó que "El estado de la cuestión es un trabajo que consiste en exponer el resultado de las investigaciones en cualquier asunto de una rama del conocimiento". Desde las características, propiedades, aspectos o dimensiones, clasificación de los objetos, personas, agentes e instituciones o de los procesos naturales o sociales con el fin de determinar e informar los modos de ser de los objetos. 
En consecuencia, el estado de la cuestión se encuentra conformado por los antecedentes del problema investigado establecidos en el momento "heurístico o de búsqueda de fuentes" (Cardoso, 2000, p.136), identificación y selección de documentos según su nivel de autenticidad y aportación teórica. Así las cosas, esta etapa se desarrolló con base en una exhaustiva revisión bibliográfica (tabla 1), con criterios de selección basados en autenticidad, trascendencia epistémica y aportes teóricos y metodológicos centrados principalmente en instituciones de formación de docentes y en las prácticas pedagógicas de maestros encargados de formar maestros. En ese sentido, se rechazaron conceptos especulativos, conjeturales e irreflexivos alejados del objetivo del presente escrito.

Tabla 1. Tipos de fuentes.

\begin{tabular}{|l|l|c|}
\hline \multicolumn{2}{|c|}{ Tipos } & Número \\
\hline \multirow{3}{*}{ Libros de investigación } & \multicolumn{1}{|c|}{ Colombia } & 19 \\
\cline { 2 - 3 } & $\begin{array}{l}\text { 1 de Australia; 1 de Brasil; 2 de España; 1 de México; 1 } \\
\text { de Perú }\end{array}$ & 6 \\
\hline \multirow{2}{*}{ Eventos } & Congreso & 1 \\
\cline { 2 - 3 } & Memorias & 3 \\
\hline Artículos & Artículos/revistas & 45 \\
\hline \multirow{3}{*}{ Tesis } & Tesis doctoral & 7 \\
\cline { 2 - 3 } & Tesis de maestría & 25 \\
\cline { 2 - 3 } & Tesis de pregrado & 1 \\
\hline Total & & 107 \\
\hline
\end{tabular}

Fuente: elaboración propia.

La búsqueda de tales antecedentes se realizó a través de fuentes de información, primarias y secundarias, halladas en la literatura, en investigaciones e indagaciones bibliográficas, hemerográficas y electrónicas, y en bases de datos como Redalyc, Researchgate, Dialnet, Google Scholar, Scielo, ScienceDirect, Teseo, Ebooks, JSTOR, Scopus, CLACSO y Web of Science, entre otras. De este modo se pudo acceder a fuentes documentales como revistas (tabla 2), artículos, informes y libros de investigación, tesis aprobadas para optar al título de doctor, magíster, especialista, licenciado o profesional y artículos de universida- des y centros de investigaciones realizados en el ámbito local, nacional o internacional. Asimismo, se abracó la diversidad en la producción académica e investigativa en áreas como educación, ciencias de la educación, historia de la educación, filosofía e historia de la educación, pedagogía, educación y psicología educativa, letras y ciencias humanas, educación y desarrollo humano, sociología de la educación, docencia, diversidad, inclusión, entre otras, que dan cuenta de los postulados que tuvieron lugar para configurar las "prácticas pedagógicas" de los maestros que forman maestros (Ñupas et al., 2014, p.386). 
Tabla 2. Revistas de investigación consultadas.

\begin{tabular}{|c|c|c|}
\hline País & Revista & Número \\
\hline \multirow{18}{*}{ Colombia } & Conversaciones Pedagógicas & 2 \\
\hline & Educación XXI & 1 \\
\hline & Educación y Humanismo & 1 \\
\hline & Memoria y Sociedad & 1 \\
\hline & Memorias & 1 \\
\hline & Nodos y Nudos & 1 \\
\hline & Nómadas & 1 \\
\hline & Pedagogía y Saberes & 3 \\
\hline & Plumilla educativa & 7 \\
\hline & Praxis \& Saber & 1 \\
\hline & Revista Colombiana de Educación & 2 \\
\hline & Revista de Investigaciones & 3 \\
\hline & Revista Educación y Ciudad & 1 \\
\hline & Revista Educación y Pedagogía & 1 \\
\hline & Revista Historia de la Educación Latinoamericana (RHELA) & 2 \\
\hline & Revista Javeriana & 2 \\
\hline & Revista Latinoamericana de Estudios Educativos & 4 \\
\hline & Revista Virtual Universidad Católica del Norte & 1 \\
\hline \multirow{3}{*}{ España } & Historia de la Educación, Revista interuniversitaria & 1 \\
\hline & Revista Iberoamericana de Educación & 1 \\
\hline & $\begin{array}{l}\text { Revista Iberoamericana sobre Calidad, Eficacia y Cambio en Educación } \\
\text { (REICE) }\end{array}$ & 1 \\
\hline \multirow{2}{*}{ México } & Perfiles Educativos & 1 \\
\hline & Reencuentro & 2 \\
\hline \multirow{2}{*}{ Brasil } & Formação Docente & 1 \\
\hline & Cadernos de História da Educação & 1 \\
\hline Perú & Helios & 1 \\
\hline Venezuela & Laurus & 1 \\
\hline
\end{tabular}

Fuente: elaboración propia.

Aunque está claro que las investigaciones presentadas de ninguna manera agotan exhaustivamente el universo epistémico del objeto de estudio, se analizó un número significativo de textos: 107 en total (tabla 3), que correspon- den a 85 textos colombianos, 9 de España, 6 de México, 3 de Brasil, 2 de Perú, 1 de Venezuela y 1 de Australia, distribuidos en 25 libros, 45 artículos científicos, 33 tesis y 4 memorias de eventos académicos. 
Tabla 3. Distribución total.

\begin{tabular}{|l|c|c|c|c|c|c|c|}
\hline & Colombia & España & México & Brasil & Perú & Venezuela & Australia \\
\hline Libro & 19 & 2 & 1 & 1 & 1 & 0 & 1 \\
\hline $\begin{array}{l}\text { Tesis doctoral } \\
\text { y maestría }\end{array}$ & 29 & 4 & 0 & 0 & 0 & 0 & 0 \\
\hline Memoria & 2 & 0 & 2 & 0 & 0 & 0 & 0 \\
\hline Revistas & 35 & 3 & 3 & 2 & 1 & 1 & 0 \\
\hline Total & 85 & 9 & 6 & 3 & 2 & 1 & 1 \\
\hline
\end{tabular}

Fuente: elaboración propia.

Este trayecto exigió, para hallar categorías internas de cada uno de los textos, la revisión, búsqueda, relación, análisis, fichaje, explicación y descubrimiento de microteorías que explican propiedades o dimensiones del objeto de estudio. Fue así como los documentos analizados, sistematizados e integrados, fueron agrupadas por focos de comprensión comunes, por títulos y temáticas, opciones metodológicas y perspectivas teóricas (Ospina y Murcia, 2012) para hacer evidente el estado de la cuestión.

Las fichas resumen hechas para todos los documentos de investigación detectados e incluidos en el presente análisis dieron lugar a las siguientes categorías emergentes: la formación del maestro: un análisis desde el discurso; prácticas pedagógicas y su relación con el quehacer del maestro; contexto histórico educativo de las escuelas normales; prácticas pedagógicas en el sistema educativo y en procesos de acreditación; y finalmente opciones metodológicas que han hecho posible el análisis de prácticas pedagógicas de maestros formadores de maestros.

\section{Formación del maestro: Un análisis desde el discurso pedagógico}

El maestro ha sido objeto de investigación desde una doble perspectiva: el deber ser de su quehacer, objetivado en la formación en instituciones formadoras de maestros, y la realidad de este, vinculada al campo declarativo, al discurso, a las voces de los maestros, a "lo dicho" en palabras de Zuluaga (1999, 41-42).

Según Larrosa (1995, p.259-329), el maestro es el resultado de su saber, su hacer y su ser. El primero, lo que sabe, tiene que ver con los discursos que definen su verdad, los conocimientos pedagógicos que tiene; el segundo, las prácticas que regulan su comportamiento, se refiere a lo que hace, su propia actividad práctica, las actuaciones explícitas en el aula, las decisiones que se toman; el tercero se relaciona con las formas de subjetividad en las que se constituye su propia interioridad, su propia manera de ser, las actitudes, los valores, las disposiciones y los componentes afectivos y emotivos.

Es así como ser maestro ha transitado, históricamente, desde una imagen "sagrada" o vocacional, con funciones altruistas fuera del aula para la comunidad, hacia una percepción secular, ocupacional, sujeta únicamente al aula, transmitida en los centros formadores de maestros (Parra, Parra y Carvajal, 1981). De este modo el maestro ha pasado a ser quien deja algunas "huellas vitales" desde su rol y quien, tras una máscara, como herramienta o como necesidad, oculta el miedo a la pérdida de control académico, del saber o del control social del aula (Garzón, Botina y Salazar, 2013). 
El maestro, especialmente de las escuelas normales superiores de Colombia, a través de la exteriorización de su consciencia colectiva intelectual, social y política (Pineda y Arbeláez, 2009), ha sido capaz de luchar, incidir y transformar la sociedad y sus procesos (Castro, 2007). Como sujeto de saber, cuenta con la pedagogía como el saber que le consagra identidad y es a quien, actualmente, se le vincula con proyectos de investigación (Bolívar y Méndez, 2013). Por tanto, el maestro es el protagonista de una "profesión de élite”, cambiante, con propuestas teóricas y prácticas que hacen que la sociedad sienta que la educación de niños y adolescentes está en buenas manos (Marina y Pellicer, 2015).

La profesión de maestro ha estado estrechamente relacionada con el conocimiento a través de la práctica pedagógica, referida a ver, hablar, pensar y adecuar el discurso de la ciencia a la edad de los sujetos del aprendizaje (De Echeverry, 1979, p.8) al vincular la teoría y la práctica, al integrar lo académico con comunidades científicas y pedagógicas (Flores, 2004) y al establecer relaciones trascendentes, de trabajo colectivo, entre la familia y la escuela (Suárez y Urrego, 2014) a través de metas y objetivos comunes, con base en necesidades de los estudiantes y del contexto de realización. En ese sentido, la profesión del docente está estrechamente relacionada con temas como la escuela y el entorno, con el sistema educativo que incluye el sistema escolar, con el éxito educativo que contiene el éxito escolar, y con la "función docente" (Marina y Pellicer, 2015).

El perfil profesional del docente es visto desde diferentes ángulos. Como instructor y como educador, resultado de la conjunción de diversas prácticas que confluyen en la escuela: las prácticas curriculares, las prácticas emergentes discursivas y las prácticas normativas (Martín, 2015). También se encuentra la visión desde la tríada creencias del maestro-práctica pedagógica-saber pedagógico, que transita constante e inseparablemente bajo el control y la influencia del habitus o estructuras incorporadas a pesar de vivir en una época determinada, con cambios culturales, y de estar inmerso en un contexto específico (Triviño, 2013).

El concepto de formación, como fenómeno vivo y como un asunto siempre presente en las normales (Taborda, 2009), ha posibilitado, desde diversas visiones pedagógicas, académicas, culturales y axiológicas, propuestas de elaboración de currículos para la formación de maestros que, tal vez, satisfagan las demandas para elaborar políticas educativas con el fin de elevar el nivel del desarrollo humano (Pineda y Loaiza, 2017; Sandoval, 2000). Es así como se ha propuesto adoptar el tema rural desde la formación inicial de los maestros, tanto en las escuelas normales como en las licenciaturas (Zamora, 2008). Asimismo, se ha planteado la urgencia de construir un nuevo paradigma que oriente el establecimiento de relaciones equilibradas entre la teoría y la práctica con aportaciones de cada una, de manera proporcional (Álvarez, 2008), de la mano de la investigación y la docencia como ejes articuladores propios de la vida académica (Bolívar y Méndez, 2013).

En este orden de ideas, se ha requerido la elaboración de un proyecto educativo propio, a partir de lo que saben realmente las instituciones sobre formación, desde las significaciones implícitas que subyacen y afectan su tarea formativa, desde la articulación del saber de la escuela con el de las comunidades (Taborda, 2009) y desde pedagogías localizadas, capaces de leer los sujetos que allí confluyen con sus necesidades y expectativas, para crear una escuela posible (Gómez y Acosta, 2006). De igual forma se han propuesto planes de estudios en instituciones formadoras de maestros, con rango universitario, basados en la tríada cultura general sólida-cultura profesional 
o de formación pedagógica y psicológica- cultura de formación de la personalidad educadora (Martín, 2015). Esta última, de manera intencionada, requiere asumir el desarrollo de competencias emocionales en los maestros en formación, en respuesta a las problemáticas y retos de la sociedad (Chica y Sánchez, 2016).

Entretanto, en el paradigma crítico-social, ha existido una distancia entre lo que se dice y lo que se hace con respecto a la formación de los maestros, vinculada con la perpetuación de modelos pedagógicos que han formado artesanos en vez de intelectuales (De Tezanos, 1986), como resultado de políticas educacionales reproductoras de turno que han exiliado la tradición pedagógica de la formación de maestros y de los contenidos curriculares (De Tezanos, 2006). Esto posiblemente ha generado, en algunas instituciones de América Latina y hasta de Europa, resultados educativos negativos en la escuela y en la sociedad y, por ende, el señalamiento y la culpabilidad a los docentes frente a la responsabilidad que los compromete en esta situación de crisis (Flores, 2004; Bonifácio, 2009). A ello se suma su poca $o$ nula preparación para la labor pedagógica a su cargo, que los deja en incapacidad de reconocer los rasgos distintivos del ejercicio rural de la educación (Zamora, 2008). En suma, como lo han manifestado algunas investigaciones, los maestros han asociado la pedagogía a la instrumentalización de la enseñanza, a procedimientos y a grandes pedagogos, evidentes en actos de aula con prácticas de enseñanza tradicionales caracterizadas por el direccionamiento y la consigna (Taborda, 2009).

\section{Prácticas pedagógicas y quehaceres del maestro}

La práctica pedagógica puede ser interpretada desde su deber ser, desde los elementos que la conforman y la originan, desde las visiones que se tienen como proceso de humanización, como quehacer del maestro, desde las exigencias que en ella se implican y desde los dos grandes paradigmas que la explican epistemológicamente: el de Zuluaga-Foucault y el de Certeau-Chartier. No obstante, también puede ser definida desde la realidad, desde los escenarios donde se materializa y legitima su ideal o su autenticidad y desde los aportes que la investigación proporciona, al estar allí inmersa.

Una categoría en la que la práctica pedagógica se hace evidente es la de los discursos, comprendidos desde la tríada identidad-disciplina-actuación, esto es, identidad del maestro según la dimensión ético-política, conceptualización según la dimensión disciplinar, y actuación a partir de las dimensiones del quehacer, lo procedimental y la estrategia (Hernández y Roncancio, 2013; Linton y Betancourt, 2014). En ese sentido, la práctica pedagógica se comprende como la relación entre el discurso, la acción y la formación pedagógica, originada en la vocación por la carrera docente, la autogestión de información y la creatividad, conjuntamente con la acción reflexiva de la praxis, la capacidad para resolver problemas y de superar dificultades, la efectividad en relaciones interpersonales en el aula y la superación de vacíos en la formación pedagógica (Vargas, 2015). Al estar expuesta a críticas y replanteamientos, colectivos e individuales, con miras a la comprensión de nuevas maneras de enseñar y nuevas maneras de aprender, esta práctica le exige al maestro identificar tendencias, paradigmas, enfoques, modelos pedagógicos y diseños de currículos desde donde podrá tomar decisiones para movilizarse y reconfigurar prácticas, habitus y creencias (Triviño, 2013).

Si se toma la práctica pedagógica como un proceso de humanización de los procesos pedagógicos, debe estar mediada por el encuentro y el reconocimiento con el otro, por la reflexión de la 
experiencia cotidiana (Taborda, Loaiza y Pineda, 2012), por la comunicación y por el respeto a la diferencia (Parra y Vallejo, 2013; Parra, Vallejo y Loaiza, 2013). Esta concepción, que logra comprenderse como emergente, ha sido gestada desde otras formas de construcción y recreación del saber adquirido que dejan huella en los estudiantes al establecer una relación menos academicista pero más humana, en la medida en que posibilitan la participación activa, la conciencia individual y la identidad propia al aceptar problemáticas y proyectos de vida (Palechor, Mera y Zúñiga, 2016).

Asimismo, la práctica pedagógica puede ser percibida en el quehacer del maestro dentro del contexto de la educación formal desde la relación dinámica, experimental y reflexiva entre la teoría y la práctica del quehacer pedagógico (Taborda et al., 2012; Hernández y Roncancio, 2013); desde las estrategias, los instrumentos y las acciones que realiza el docente en el aula de clase (Loaiza, Duque y Vallejo, 2013; Zambrano, Rivera, Fernández y González, 2014); desde sus propias dimensiones y desde las diferentes tradiciones que la han orientado (Linton y Betancourt, 2014).

Cabe recordar que la práctica pedagógica depende del contexto social, de la personalidad del docente, de las características de los estudiantes, del tipo de persona, sociedad y cultura que la institución quiere formar, del uso de estrategias, técnicas, actividades y acciones pedagógicas, de estructuras curriculares (Valencia, 2013) y de las características u orientaciones de las prácticas sociales que asignan unas funciones a los sujetos de esa práctica (De Echeverry, 1979; Zuluaga, 1999). Es así como la práctica pedagógica se comprende como espacio, como una apuesta política, social y cultural de reivindicación de las relaciones con el territorio y los procesos culturales (Ruiz, Ortiz y Soler, 2013).
Más aún, la práctica pedagógica se ve afectada por la historia de vida tanto del actuar diario como del actuar en el aula, en la comunidad y en las relaciones que se entretejen con los demás. Por ello es vista y comprendida como huella vital (Burbano y Cortez, 2013; López y Pereira, 2014), como aprendizaje en lecciones de vida (Pereira, López y Ríos, 2015), como configuración desde la autoevaluación institucional (Vargas, 2016), o como relación con prácticas de vida (Mora, Caicedo y Mejía, 2013), con el miedo (Garzón et al., 2013), con la diversidad (Gómez, Guerrero y Buesaquillo, 2013; Kremer y Quijano, 2015), con la diversidad en procesos lectoescriturales (Samboni, Rengifo, y Valencia, 2015), con la diversidad desde la dimensión ambiental (Campo, Salazar, Valdés y Jiménez, 2014), con la innovación (Achipíz, Perdomo, Chicangana y Jojoa, 2016; Gómez, Muñoz y Cadavid, 2015), con la transformación (Navia, Navia y Timaná, 2015), con experiencias significativas (Calvache, Ruiz, Tombé y Roa, 2016), con la convivencia escolar (Erazo y Obando, 2016) y con las políticas de inclusión educativa (Perdomo, Herrera, Virguez y Garzón, 2016).

La práctica pedagógica, además, ha sido vista desde dos miradas epistemológicas diferentes que deben ser reflexionadas por tener ciertos puntos de encuentro que podrían favorecer el quehacer del maestro. Por un lado, ZuluagaFoucault la conciben como una práctica pedagógica estructural, con dispositivos y formatos escriturales de las ciencias, que recoge las teorías pedagógicas institucionales o alternativas, los saberes no formales, los saberes populares y los locales para hacer generalizaciones estructurales sobre la escuela y que van mucho más allá de lo que el maestro hace en el aula, en el salón. Por otro lado, Certeau-Chartier apunta a la cotidianidad contingente, a la experiencia más inmediata de los maestros, a la noción de "artes de hacer", con tácticas sin discurso que evidencian 
singularidades, cotidianidad, diferencias culturales de una situación a otra, múltiples prácticas diseminadas y procedimientos infinitesimales que no han sido instituidos ni formalizados por estrategias ni por "mallas de los poderes" legislativo-normativas (Saldarriaga, 2016, p.19).

A la par, se ha reconocido que, entre los modelos pedagógicos normalizador o tradicional, academicista, técnico-eficientista, humanista o personalista y hermenéutico-reflexivo, el primero es el que prevalece como aquel que ha estructurado la práctica pedagógica de los maestros. Así las cosas, el modelo tradicional representó el eje central de la formación en la mayoría de las investigaciones analizadas en cuanto a ideas, valores y metas pedagógicas de los docentes en ejercicio (Flórez y Batista, 1982). Esto se evidencia en las propuestas curriculares (Pineda y Loaiza, 2017), con áreas fraccionadas, sin transversalización de saberes y poco acordes con las necesidades de los estudiantes (Parra y Vallejo, 2013; Parra et al., 2013), igualmente justificadas en las actitudes y expresiones de los maestros de resistencia al cambio en sus prácticas de aula (Trujillo, 2016) y en la transmisión, fijación y acumulación de conocimientos teóricos como eje central del modelo (Duque, Vallejo y Rodríguez, 2013; Loaiza et al., 2013).

Esta última concepción de práctica pedagógica, en contrasentido con lo que se conceptualiza, confirma la distancia existente entre los discursos, enmarcados en teorías pedagógicas edificadas sobre procesos iniciales de formación docente, y las actuaciones o el quehacer del maestro, lo práctico o el mundo cotidiano (Bolaño, 2014), que continúa anclado en concepciones tradicionales y tecnicistas que repiten modelos de actuación de sus profesores al acudir a sus experiencias como estudiantes (Hernández y Roncancio, 2013). Este fenómeno se manifiesta en el maestro por la poca reflexividad de las experiencias que se originan cuando el conocimiento está en circulación, dada la desarticulación entre el saber disciplinar y el pedagógico (Luna y Haydar, 2012), o cuando existen inconsistencias al intentar acomodar su discurso a las exigencias del medio o de la institución donde laboran, sobre todo en lo que respecta a la aplicación de nuevas tendencias y metodologías (Linton y Betancurt, 2014).

Por otra parte, existen prácticas pedagógicas facilitadoras del aprendizaje, donde se origina una relación entre lo que los maestros dicen y hacen (Linton y Betancurt, 2014), con capacidad de gestión social y cultural desde su accionar pedagógico, en contextos rurales y urbanos marginales (Aguilar, 2011). Simultáneamente, existe un número menor de maestros que emplean el aprendizaje colaborativo, el aprendizaje basado en problemas, el aprendizaje por investigación y el aprendizaje por discusión o debate, al tiempo que recurren a técnicas como la lúdica, el taller y los mapas conceptuales (Valencia, 2013). En todo caso, dadas las condiciones de la cultura escolar actual, la institucional y la académica, se afirma que, especialmente para los maestros practicantes, es difícil entrar en una institución educativa con propuestas pedagógicas diferentes, flexibles, basadas en la experiencia de ellos mismos que logren romper con prácticas escolares de tradición (Ruiz et al., 2013).

A lo largo del siglo $\mathrm{XX}$, especialmente en Norteamérica, la investigación educativa ha estado determinada desde instancias internas de la institución, donde los profesores han sido los investigadores principales, impulsados por psicólogos y psicométricos, y desde instancias externas por parte de investigadores pertenecientes a las ciencias sociales como sociólogos, antropólogos y analistas del comportamiento (LeCompte, 2009). En Colombia, solo hasta en el siglo XXI se ha incursionado con fuerza en investigaciones educativas donde instituciones 
formadoras de maestros como las escuelas normales e instituciones universitarias, así como los sujetos y sus discursos, han sido los ejes centrales. De esta manera se ha ido posicionando la figura del maestro como investigador y como profesor indagador, en relación con los procesos pedagógicos y la escuela (Ossa, 2015).

Sin embargo, se ha demostrado que tal posicionamiento del maestro ha sido únicamente un asunto de aceptación general, con poca apropiación y claridad en procesos investigativos, con esporádica presencia de informes de investigación producto del hacer cotidiano (Castaño, 2008), lo cual se evidencia en la escasez investigativa en temas relacionados con la formación docente y pedagógica. Esta situación, tal vez, se ve propiciada por la poca incentivación, inversión económica y financiación para la investigación (Bolaño, 2014) o por la falencia en la cultura de la investigación como ejercicio operativo impuesto sin previa formación al capital humano. Es así como expertos investigadores recomiendan la estructuración y conformación de un grupo de académicos críticos y de redes de trabajo colaborativo, capaces de avanzar hacia la consolidación de la cultura de la investigación desde las prácticas pedagógicas investigativas en las escuelas normales superiores, con base en realidades sociales que consideren la calidad como un proceso altamente formativo y objetivo (Saker, 2014).

\section{Contexto histórico educativo de las escuelas normales}

Para comprender las prácticas pedagógicas de los maestros formadores de maestros, se debe vislumbrar primero la perspectiva temporal que gestó tal o cual suceso o fenómeno. Esta es una condición necesaria para aprehender el desarrollo de sistemas dados e identificar las interconexiones que indican sus papeles respectivos en el proceso de la historia, tiempo concebido como múltiple o diverso que puede y debe ser tomado en cuenta en toda investigación (Cardoso, 2000, p.194, 213). Es por ello que, como lo afirma Cerda (2011, p.145-150), se hace necesario ampliar el campo de la materia histórica de archivos y fuentes documentales a la actividad humana en su conjunto, a las voces de los protagonistas, e incluir el análisis científico de las relaciones existentes entre los diversos procesos y componentes que han participado en las grandes formaciones y transformaciones sociales, económicas, técnicas y culturales de la humanidad.

Es así como, específicamente, las escuelas normales como instituciones formadoras de maestros para la educación básica han sido objeto de estudio de diversas investigaciones, tanto nacionales como internacionales, que las han reflexionado y hasta problematizado a fondo. Así se ha abordado su génesis con base en perspectivas pedagógicas y político-sociales, a lo largo del siglo XIX (Báez, 2004b; Seraphim y Lima, 2018; Souza, Gonçalves y Pádua, 2008), hasta académicas y administrativas relacionadas con sus dinámicas histórico-económicas y político-religiosas que implicaron vicisitudes en el sistema educativo en la transición hacia la primera mitad del siglo XX (Helg, 2001; Valencia, 2006; Pinto y García, 2003). También se ha abordado el impacto educativo y cultural en la formación de maestros (Herrera y Low, 1994; Pineda, 2007) a lo largo de la segunda mitad del siglo XX (Benejam, 2002; Cárdenas, 2002; Ríos y Cerquera, 2013) y de las incursiones de modelos pedagógicos foráneos en el rol del maestro formador de maestros que por ende afectaron a estas instituciones (Loaiza, 2009). Así pues, se ha observado que las escuelas normales, dada su vinculación con facultades de Educación de universidades pedagógicas (Farid, 2015; Palomero, Fernández y Teruel, 2008) y de movimientos pedagógicos, nacionales y regionales (Loaiza, Pineda y Arbeláez, 2014), posibilitaron formas de saber y poder en la práctica 
pedagógica de los maestros normalistas (López, 2009) que perduran aún en el siglo XXI (Calvo, Rendón y Rojas, 2004).

\section{Contexto educativo y proceso de acreditación}

Las escuelas normales son reconocidas como instituciones formadoras de maestros para el nivel de educación que, según el nivel propedéutico, haya asumido cada país para formar o bien tecnólogos en educación o bien profesionales licenciados de nivel superior. En América Latina, la mayoría de ellas han desaparecido, como en el caso de Brasil (Souza et al., 2008) o Chile; algunas se han transformado hacia el nivel superior de las facultades de Educación, como en el caso de México o Bolivia; y otras aún permanecen en el mismo nivel, como en el caso colombiano. Sin embargo, en los dos últimos casos han estado constantemente evaluadas, tanto por organismos internos propios del sistema educativo como por organismos externos, internacionales, que así lo han recomendado.

Desde la década del setenta del siglo XX, las evaluaciones de las instituciones formadoras de maestros y de instituciones de educación en Colombia ya evidenciaban la reducción de la relación pedagógica entre profesor y alumnomaestro a indicadores formales (Briones, 1978). En estos influían variables externas e internas en el proceso de enseñanza-aprendizaje con el fin de juzgar acerca de la efectividad y calidad del sistema escolar primario y secundario (Restrepo, Villalobos, Ospina y Buitrago, 1981).

En este rubro se destaca el informe del Ministerio de Educación Nacional colombiano (MEN) para las escuelas normales del país, donde se afirmó que solo el $24,2 \%$ de estas tenía calidad apropiada para la enseñanza y se sugirió suprimir el 75,8 \% a partir de 1979 (Rátiva, 2015). Tres años más tarde, dichas normales se convirtie- ron en bachillerato pedagógico hasta principios del siglo XXI, cuando pasaron a reestructurarse (Messina, 1999) y denominarse escuelas normales superiores. Estas contaron así con un único sistema para la formación de maestros de educación preescolar y básica primaria, con dos años de escolaridad posteriores al bachillerato pedagógico, y fueron integradas en redes regionales. También fueron reformadas las facultades de Educación de las universidades oficiales y supervisadas por las Secretarías de Educación de los departamentos (Müller, 1992).

En estos nuevos esquemas se insertaron prácticas profesionales que se asumieron como un momento en la formación del docente, como un ejercicio de experimentación, diseño y ejecución de experiencias pedagógicas que forman parte del proceso formativo y que buscan el desarrollo de competencias, habilidades, conocimientos y teorías que se ponen en funcionamiento durante la enseñanza-aprendizaje (Rátiva, 2015).

Entretanto, para el caso boliviano, la transformación curricular, estructural, institucional y pedagógica en la formación de profesores de nivel primario igualmente fue posible gracias al Programa de Reforma Educativa 1995-1999. De este modo se reconvirtieron las 19 escuelas normales en institutos normales superiores, adscritos a universidades mediante el diseño curricular basado en 26 competencias y en la adopción de nuevas estrategias como el enfoque integrado de la práctica pedagógica planificada con investigación en el aula, trabajo en equipo y atención a la diversidad social (Barrera, 2007). Por otra parte, para el caso mexicano, a lo largo del siglo XX se intentó profesionalizar la formación del magisterio, pero solo hasta finales de dicho siglo lapso se consideró formar profesionales académicos con el perfil de profesor-investigador a través del programa para la transformación de las escuelas normales y de las universidades pedagógicas, con el apoyo 
de cuerpos académicos, los cuales son considerados como la unidad básica del Programa para el Mejoramiento del Profesorado (Vera, 2011). Este programa ha contemplado desde 2001 hasta la fecha la planeación y evaluación institucional, interna y externa, como criterio para mejorar la organización y el funcionamiento de las normales, así como el trabajo colaborativo entre pares para la investigación como un elemento detonador del proceso de calidad (Gutiérrez, 2006).

Finalmente, expertos investigadores manifiestan la necesidad de diseñar políticas asociadas a los planes decenales de educación para cada una de las etapas que se proponen de formación de un maestro: desde la elección de su carrera y la formación inicial hasta la formación en servicio y la carrera docente. Asimismo, se sugiere estudiar las instituciones de formación por dentro, desde las investigaciones que ellas realizan —sus temas, problemas y métodos-, para analizar su relación con la profesionalidad del docente, en una época donde existe gran oferta de licenciaturas, presenciales, a distancia y virtuales (Calvo, 2007). Otra recomendación es recoger la experiencia de la acreditación de calidad y desarrollo de estas instituciones en los aspectos que garantizan la transformación efectiva de las prácticas de formación y que han dado lugar a experiencias significativas en la búsqueda de respuestas cada vez más acordes con las necesidades de la infancia y gestoras a su vez de otras formas de existencia (Baracaldo, 2007). El fin de esto último es sistematizar y reflexionar en torno al saber pedagógico para replantear el Proyecto Educativo Institucional (PEI) y el Programa de Formación Complementaria (PFC) (Granados, 2014).

\section{Opciones metodológicas: maestros formadores de maestros}

Son diversas las opciones metodológicas que se resaltan de los textos analizados. Estos se centran especialmente en tres ámbitos: la recuperación histórica de las instituciones, especialmente para las escuelas normales, con el método histórico; la archivística y la recuperación de archivos históricos y fuentes primarias; y las biografías y autobiografías de los sujetos docentes para reflexionar frente a su experiencia pedagógica y constituir un saber pedagógico.

La recuperación histórica de las escuelas normales ha sido un tema de gran interés tanto en América Latina, en países como México (Arteaga y Camargo, 2014; Beltrán y Berrelleza, 1998), Brasil (Lima, 2010; Seraphim y Lima, 2018), Argentina (Chaile, 2004) y Colombia (Báez, 2004a; Herrera y Low, 1994; Loaiza, 2009; Loaiza et al., 2014; López, 2009; Valencia, 2006), como en Europa, especialmente en España (Ramírez, 2006). Ha sido desde el método histórico y las fuentes primarias, documentales y orales que la comprensión y explicación de lo ocurrido, a lo largo de la existencia de estas instituciones, se ha dado a conocer para conservar las raíces, esclarecer los sentidos y perspectivas de las entidades y fortalecer los lazos de identidad gremial y social (Beltrán y Berrelleza, 1998).

Dentro de las tendencias metodológicas, en los textos analizados prevalece el paradigma cualitativo en las investigaciones educativas con múltiples diseños como el descriptivo (Loaiza et al., 2013) y el descriptivo comprensivo (Parra et al., 2013), desde varios enfoques como el interpretativo (Hernández y Roncancio, 2013; Palechor et al., 2016), el histórico hermenéutico (Pineda y Loaiza, 2017; Pradilla, 2013), la historia de las mentalidades (Ramírez, 2006), la complementariedad etnográfica (Suárez y Urrego, 2014) y la investigación-acción educativa (Ruiz et al., 2013). Sumado a ello, las técnicas e instrumentos de recolección de información están acordes con el paradigma cualitativo, desde fuentes documentales (Lima y Seraphim, 2016; Loaiza et 
al., 2014), archivos y fuentes primarias (Arteaga y Camargo, 2012; Martín, 2015), entrevistas (González, Jiménez, Neira y Cortés, 2015), entrevistas narrativas (Trujillo, 2016), entrevistas semiestructuradas (Duque et al., 2013), entrevistas a profundidad (Garzón et al., 2013), grupos focales (Valencia, 2013), autobiografías (Triviño, 2013), autobiografías narrativas (Parra y Castañeda, 2014), historias de vida (Rátiva, 2015; González et al., 2015), observación de clase (Linton y Betancurt, 2014), observación participante (Zambrano et al., 2014) y observación directa (Luna y Haydar, 2012).

Las fuentes de información se centraron principalmente en profesores de primaria y secundaria, en profesores de escuelas normales superiores (Loaiza, 2009; Pineda y Arbeláez, 2009), en docentes de facultades de Educación, en directivos docentes, en egresados de instituciones formadoras de maestros, en estudiantes de facultades de Educación, en estudiantes de Ciclo de Formación Complementaria (CFC) (Baracaldo, 2011) y de Programa de Formación Complementaria (PFC). El análisis y procesamiento de información principalmente giró en torno al análisis documental (Aguilar, Yarza y Paredes, 2007; Granados, 2014) con Atlas.ti (Vargas, 2015), al análisis de contenido (Bolívar y Méndez, 2013) y a la interpretación, comprensión y evaluación de los datos que se extrajeron, organizaron y estructuraron progresivamente (Chaile, 2004).

\section{CONCLUSIONES}

Las escuelas normales, como instituciones formadoras de maestros, han sido objeto de estudio de diversas investigaciones, tanto nacionales como internacionales, que las han reflexionado desde sus inicios en el siglo XIX hasta la actualidad. En este sentido se han abordado aspectos tan importantes como su historia pedagógica, de formación, de saber pedagógico, académica y administrativa, tanto en el interior de las instituciones como en la relación externa con el sistema educativo, a partir de dinámicas económicas, políticas y religiosas que las han modificado, reestructurado, acreditado e impactado en la formación de maestros, en el rol del formador de maestros y en las prácticas pedagógicas.

El maestro por su parte también ha sido objeto de investigación, tanto desde el deber ser de su quehacer - con base en la formación ofrecida e implantada por las instituciones formadoras de maestros, llámense escuelas normales o facultades de Educación - como desde la realidad de la profesión, vinculada al campo declarativo, a las voces de los maestros, a la experiencia donde se confronta la gran distancia entre lo que el docente dice y lo que hace.

El quehacer del maestro, sujeto del saber pedagógico de las escuelas normales superiores y facultades de Educación, es otro elemento que ha sido analizado. En este caso se contemplan tres dimensiones que conjuntamente regulan el escenario pedagógico, el quehacer cotidiano: el saber, caracterizado por un conocimiento pedagógico, didáctico y curricular evidenciado en lo declarativo en el discurso; el hacer en lo procedimental, es decir, en la práctica pedagógica, en las experiencias propias y foráneas, en las reflexiones en la práctica y sobre la práctica pedagógica, en la planeación y en la acción; y el ser.

La práctica pedagógica se ve definida como el discurso, la acción y la formación pedagógica, arbitrada entre lo que el maestro debería saber, según los elementos constitutivos de la práctica, y la visión como proceso de humanización que viabiliza el quehacer del maestro, con base en las exigencias que la direccionan. Se trata de una práctica pedagógica que, en la realidad de 
los escenarios pedagógicos, experimenta, materializa y legitima su ideal o su autenticidad, de la mano de los aportes que la reflexión y la investigación pedagógica le proporcionan al maestro al concientizarlo frente a la dicotomía entre sus discursos y sus actuaciones o quehaceres. De este modo al docente se le posibilita problematizar las prácticas pedagógicas que, tal vez, le ayuden a avanzar hacia la consolidación de la cultura de la investigación desde las prácticas pedagógicas investigativas.

Las escuelas normales de algunos países de América Latina han sido evaluadas tanto por organismos internos propios del sistema educativo de cada país como por organismos externos, internacionales, cuyas recomendaciones han llevado a la reestructuración y acreditación de tales instituciones con miras a alcanzar altos índices de calidad. Tal reestructuración les ha exigido pensarse desde elementos como la pedagogía, la formación de maestros, el saber pedagógico y las prácticas pedagógicas de los maestros de cara a su misión de formar o bien tecnólogos en educación, también llamados maestros para el nivel de educación básica primaria, o bien en el nivel superior, con la formación de licenciados para la educación secundaria y media.

Los escenarios que más se privilegiaron en los procesos de práctica pedagógica de maestros encargados de formar maestros, bien para la educación preescolar y básica primaria o bien para la básica secundaria, media o universitaria, fueron aquellos que propiciaron ambientes de enseñanza y de aprendizaje como el aula y la institución educativa, donde todavía impera el modelo tradicional, aunque el escenario social fuera del aula, como espacio pedagógico donde la comunidad cumplió un rol protagónico, fue importante. Las actividades que más se desarrollan dentro de la práctica pedagógica se centran en lo académico y evaluativo, con algunos tintes sociales asignados a la función del quehacer del maestro, haciendo evidente aún la distancia entre el discurso y el quehacer pedagógico.

Los teóricos que predominan al hablar de práctica pedagógica son Loaiza (Loaiza et al., 2012; Loaiza et al., 2013; Loaiza et al., 2014; Loaiza y Duque, 2017), con la tríada discurso-acciónescenario pedagógico; y Martínez (1990), Zuluaga (1999) y De Echeverry (1979), con sus investigaciones en el campo educativo frente a la práctica pedagógica y el discurso pedagógico. En la categoría discurso pedagógico, se destacan los postulados de Díaz (1995), de la mano del sociólogo Basil Bernstein. Finalmente, en la categoría de acción pedagógica se encuentran De Tezanos $(1986,2006,2015)$ y Herrera y Low (1994) con el quehacer de los maestros, así como Larrosa (1995) y Schön (1998), con la experiencia y el profesional reflexivo.

Son diversas las opciones metodológicas que se resaltan de los 107 textos analizados. Entre ellas ha prevalecido el paradigma cualitativo en investigaciones educativas, en su gran mayoría de tipo comprensivo, desde enfoques interpretativos, histórico-hermenéuticos, con fuentes documentales, archivos y fuentes primarias, entrevistas, grupos focales, autobiografías, historias de vida, observación de clase, observación participante y observación directa. Esto evidencia la necesidad cada vez más urgente de descifrar la realidad pedagógica del maestro, de desentrañar justificadamente la toma de decisiones intencionadas por parte de este actor.

Finalmente, dentro de los limitantes para alcanzar el propósito del presente artículo se manifiestan las restrictas y hasta carentes descripciones metodológicas de algunos de los textos estudiados frente a temas trascendentales como la opción metodológica, el diseño de investigación, las técnicas e instrumentos de recolección de 
información o interpretación de esta, los actores protagonistas y los focos de estudio, entre otros. Esta circunstancia dificultó el acercamiento reflexivo a tales referentes; por lo tanto, se recomienda hacer explícitos estos aspectos dentro de los textos académicos para permitir profundos acercamientos al estado de la cuestión de un objeto de estudio determinado.

\section{REFERENCIAS BIBLIOGRÁFICAS}

Achipíz, Ch. C., Perdomo, C. A., Chicangana, N. D. y Jojoa, J. M. (2016). Huellas vitales e innovación. Plumilla Educativa, (17), 34-52.

Aguilar, D. A., Yarza, R. A. y Paredes, D. M. (2007). Archivo pedagógico normalista como un campo aplicado. Aproximaciones para la formación de maestros y la investigación pedagógica en las Escuelas Normales Superiores. Pedagogía y Saberes, (26), 87-96.

Aguilar, R. D. (2011). Los egresados normalistas en el departamento de Antioquia: institución formadora, maestros y región. Nodos y Nudos, 3(30), 65-78.

Álvarez, A. C. (2008). La relación teoría práctica en la enseñanza y el desarrollo profesional del docente (tesis doctoral). Universidad de Oviedo, España.

Arteaga, C. B. y Camargo, A. S. (12 de marzo de 2012). Los archivos históricos de las escuelas normales y la historia ignota de la formación de docentes en México. En H. Casanova (Presidencia), Historia e Historiografía de la Educación. Ponencia llevada a cabo en el XI Congreso Nacional de Investigación Educativa. México, Ciudad de México.

Arteaga, C. B. y Camargo, A. S. (2014). Organización de los archivos históricos de las escuelas normales de México y el aporte de su contenido a la historia de la educación. Perfiles Educativos, 36(145), 157-174.

Báez, O. M. (2004a). Las escuelas normales de varones del siglo XIX en Colombia. Revista Historia de la Educación Latinoamericana, RHELA, 6(6), 179-208.
Báez, O. M. (2004b). Las escuelas normales y el cambio educativo en los Estados Unidos de Colombia en el periodo radical, 1870-1886. Tunja, Colombia: Universidad Pedagógica y Tecnológica de Colombia.

Baracaldo, Q. M. (2007). Escuelas normales superiores en el marco de la Visión 2019. Revista Javeriana, 74(733), 38-45.

Baracaldo, Q. M. (2011). La subjetividad en la formación de maestros. Nómadas, (34), 246-259.

Barrera, A. S. (2007). El desarrollo de competencias y los factores innovadores integrados en la formación docente inicial: una experiencia en institutos normales superiores de Bolivia. Revista Iberoamericana sobre Calidad, Eficacia y Cambio en Educación, REICE, 5(5), 81-91.

Beltrán, L. D. y Berrelleza, F. M. (1998). La Escuela Normal de Sinaloa: una visión histórica. Culiacán Rosales, Sinaloa, México: Escuela Normal de Sinaloa.

Benejam, A. P. (2002). Las escuelas normales en tiempos de la transición (principios de siglo XX hasta la ley general de educación de 1970). Revista Interuniversitaria, (21), 81-90.

Bolaño, G. Y. (2014). Estado del arte sobre las investigaciones de prácticas pedagógicas de los maestros en formación en universidades colombianas en el periodo de 2010 al 2013 (tesis maestría). Universidad de San Buenaventura, Bogotá.

Bolívar, M. J. y Méndez, G. S. (2013). La formación de maestros y su relación con la investigación, en las publicaciones seriadas de la Universidad Pedagógica Nacional (tesis pregrado). Universidad Pedagógica Nacional, Bogotá.

Bonifácio, C. da S. (2009). Professores e escolas, a imagem social do professor do ensino básico no Portugal contemporáneo (1973-2005) (tesis doctoral). Universidad de Salamanca, España.

Briones, G. (1978). Evaluación de las escuelas normales de Colombia. Revista Colombiana de Educación, 2(2), 95-98.

Burbano, C. Y. y Cortez, L. I. (2013). Prácticas pedagógicas y huellas vitales (tesis maestría). Universidad de Manizales, Colombia. 
Calvache, H. E., Ruiz, V. J., Tombé, T. M. y Roa, D. Z. (2016). Experiencias significativas y huellas vitales (tesis maestría). Universidad de Manizales, Colombia.

Calvo de, M. G. (2007). La pregunta por la profesionalidad del docente. Revista Javeriana: $E l$ Pensamiento Cristiano En Diálogo Con El Mundo, 74(733), 26-37.

Calvo, G., Rendón, L. D. y Rojas, L. (2004). La formación de los docentes en Colombia. Estudio diagnóstico. Colombia. Universidad Pedagógica Nacional, Organización de las Naciones Unidas para la Educación, la Ciencia y la Cultura (UNESCO), Instituto Internacional para la Educación Superior en América Latina y el Caribe (IESALC). Bogotá, Colombia: Universidad Pedagógica Nacional.

Campo, D. B., Salazar, M. J., Valdés, A. F. y Jiménez, C. P. (2014). Prácticas pedagógicas en el contexto de la diversidad de los sujetos, una mirada desde la dimensión ambiental (tesis maestría). Universidad de Manizales, Colombia.

Cárdenas, P. Y. (2002). Tensiones de identidad durante la segunda mitad del siglo XX en Colombia: aproximaciones desde la producción de textos escolares de los egresados de la Escuela Normal Superior Nacional (tesis maestría). Universidad Pedagógica Nacional, Bogotá.

Cardoso, S. C. (2000). Introducción al trabajo de la investigación histórica, conocimiento, método e historia. Barcelona, España: Crítica.

Castaño, D. G. (2008). ¿Investigación en las escuelas normales? Conversaciones Pedagógicas, (6), 113-122.

Castro, V. J. (2007). Maestro: condición social y profesión docente en Colombia, 1991-2002. Bogotá, Colombia: Universidad Pedagógica Nacional, Instituto para la Investigación Educativa y el Desarrollo Pedagógico.

Cerda, G. H. (2011). Los elementos de la investigación: cómo reconocerlos, diseñarlos y construirlos. Bogotá, Colombia: Magisterio.

Chaile, M. O. (2004). Configuración de la formación docente de los niveles básico y medio, en la provincia de Salta, Argentina, en relación con la función de Estado (1950-1995) (tesis doctorado). Universidad Rovira y Virgili, España.

Chica, P. O. y Sánchez, B. J. (2016). Educación y pedagogía: un debate en movimiento en perspectiva de la transformación social y sus implicaciones para las organizaciones educativas. En Ramírez M. G. (Comp.), El Análisis Organizacional en México y América Latina, retos $y$ perspectivas a 20 años de estudio. (volumen I) (pp. 305-346). México: HESS.

De Echeverry, O. L. (1979). Hacia una historia de la práctica pedagógica colombiana. Enfoque conceptual básico del proyecto. Revista Colombiana de Educación, 4(2), 1-9.

De Tezanos, A. (1986). Los maestros como artesanos intelectuales, un estudio crítico sobre la formación. Bogotá, Colombia: Centro de Investigaciones, Universidad Pedagógica Nacional.

De Tezanos, A. (2006). El maestro y su formación, tras las huellas y los imaginarios. Colección Pedagogía e Historia. Bogotá, Colombia: Magisterio.

De Tezanos, A. (2015). Oficio de enseñar-saber pedagógico: la relación fundante. Educación $y$ Ciudad, (12), 7-26.

Díaz, V. M. (1995). Aproximaciones al campo intelectual de la educación. En J. Larrosa. (Ed.), Escuela, poder y subjetivación (pp. 333-361). Madrid, España: La Piqueta.

Duque, P. A., Vallejo, A. S. y Rodríguez, R. J. (2013). Prácticas pedagógicas y su relación con el desempeño académico (tesis maestría). Universidad de Manizales - Fundación Centro Internacional de Educación y Desarrollo Humano Cinde, Colombia.

Erazo, C. C. y Obando, V. V. (2016). Las huellas vitales y su impacto en la renovación de las prácticas pedagógicas frente a la convivencia escolar (tesis maestría). Universidad de Manizales, Colombia.

Farid, R. M. (2015). Escuelas normales y formación de educadores desde una educación para niños con discapacidad. Revista Historia de la Educación Latinoamericana, RHELA, 17(25), 35-50. 
Flores, A. I. (2004). ¿Cómo estamos formando a los maestros en América Latina? Lima, Perú: Programa de Educación Básica de la Cooperación Alemana al Desarrollo (PROEDUCA), GTZ, Oficina Regional de Educación de la UNESCO para América Latina y el Caribe.

Flórez, O. R. y Batista, J. E. (1982). El pensamiento pedagógico de los maestros de educación primaria oficial de Medellín. Medellín, Colombia: Copiyepes.

Garzón, A. M., Botina, P. M. y Salazar, B. J. (2013). El miedo en las prácticas pedagógicas (tesis maestría). Universidad de Manizales, Colombia.

Gómez, M. A. y Acosta, J. W. (2006). Diversidad cultural en la formación de maestros. Bogotá, Colombia: Universidad Pedagógica Nacional, Red de Formación Docente de América Latina y del Caribe, Kipus.

Gómez, O. E., Guerrero, M. G. y Buesaquillo, B. M. (2013). Prácticas pedagógicas y diversidad (tesis maestría). Universidad de Manizales, Colombia.

Gómez, U. M., Muñoz, C. F. y Cadavid, M. A. (2015). Huellas vitales e innovación en las prácticas pedagógicas. Plumilla Educativa, (16), 105-127.

González, L. M., Jiménez, B. A., Neira, U. A. y Cortés, S. R. (2015).Historia-memoria. Alternativasformación maestros. Convenio de Cooperación Internacional: Instituto para la Investigación Educativa y el Desarrollo Pedagógico (IDEP) y el Instituto para el Desarrollo y la Innovación Educativa (IDIE) de la Organización de Estados Iberoamericanos (OEI). Bogotá, Colombia: Magisterio.

Granados, R. D. (2014). Prácticas de enseñanza en la Escuela Normal Superior de Amaga 19972012 (tesis maestría). Universidad de Antioquia, Medellín.

Gutiérrez, L. C. (2006). El mejoramiento institucional de las escuelas normales en el gobierno del cambio. Reencuentro, (45), 1-17.

Helg, A. (2001). La educación en Colombia, 19181957: una historia social, económica y política. Serie Educación y Cultura. Bogotá, Colombia: Universidad Pedagógica Nacional.
Hernández, E. M. y Roncancio, S. P. (2013). Las concepciones de práctica pedagógica de las estudiantes de licenciatura en pedagogía infantil con alto y bajo desempeño en la práctica (tesis maestría). Universidad Tecnológica de Pereira, Colombia.

Herrera, C. M. y Low, P. C. (1994). El caso de la escuela normal superior: los intelectuales y el despertar cultural del siglo: una historia reciente y olvidada. Bogotá, Colombia: Universidad Pedagógica Nacional.

Kremer, M. S. y Quijano, V. O. (2015). Prácticas pedagógicas, promoción de inclusión y diversidad en una institución educativa de Popayán. Plumilla Educativa, (15), 67-86.

Larrosa, J. (1995). Tecnologías del yo y educación. Notas sobre la construcción y la mediación pedagógica de la experiencia de sí. En J. Larrosa. (Ed.), Escuela, poder y subjetivación (pp. 259-329). Madrid, España: La Piqueta.

LeCompte, M. D. (2009). Trends in Research on Teaching: An Historical and Critical Overview. En J. S. Lawrence. (Ed.). International Handbook of Research on Teachers and Teaching (pp. 25-60). Australia: Springer.

Lima, J. J. (2010). Formação de professores na América: notas sobre história comparada da educação no século XX. Formação docente, 02(02), 54-67.

Lima, J. J. y Seraphim, P. J. (2016). A Escola Normal de Ouro Preto - um percurso marcado por crises e reestruturações (1835-1852). Cadernos de História da Educação, 15(2), 679-699.

Linton, T. B. y Betancourt, A. M. (2014). Las concepciones de práctica pedagógica de maestros en ejercicio de la institución educativa El Pital del municipio de Pereira (tesis maestría). Universidad Tecnológica de Pereira, Colombia.

Loaiza, Z. Y. (2009). Sabio o erudito: el maestro de las escuelas normales del departamento de Caldas 1963-1978. Libros de Investigación N. 33. Manizales, Colombia: Universidad de Caldas. 
Loaiza, Z. Y. y Duque, P. A. (2017). Contexto de las prácticas pedagógicas de los maestros y los docentes. Plumilla Educativa, 19(1), 60-78.

Loaiza, Z. Y., Duque, P. A. y Vallejo A. S. (2013). Caracterización de las prácticas pedagógicas de los docentes universitarios en el área de la salud. Revista de Investigaciones, 13(22), 22-34.

Loaiza, Z. Y., Pineda, R. Y. y Arbeláez, J. N. (2014). Debate pedagógico: el maestro de las escuelas normales de Manizales en el contexto del movimiento pedagógico colombiano. Manizales, Colombia: Universidad de Caldas.

Loaiza Z. Y., Rodríguez, R. J. y Vargas, L. H. (2012). La práctica pedagógica de los docentes universitarios en el área de la salud y su relación con el desempeño académico. Revista Latinoamericana de Estudios Educativos, 8(1), 95-118.

López, G. M. (2009). Formas de saber y poder en la práctica pedagógica de los maestros normalistas, un estudio histórico (tesis doctorado). Rudecolombia, Manizales, Colombia.

López, C. F. y Pereira, A. G. (2014). Las huellas vitales y sus prácticas pedagógicas en el área industrial del Politécnico Colombiano Jaime Isaza Cadavid (tesis maestría). Universidad de Manizales, Colombia.

Luna, D. J. y Haydar, M. M. (2012). Saber del maestro de la Escuela Normal Superior Cartagena de Indias y sus implicaciones en la práctica pedagógica (tesis maestría). Universidad de San Buenaventura, Bogotá, Colombia.

Marina, J. A. y Pellicer, M. J. (2015). Libro blanco de la profesión docente y su entorno escolar. España: Ministerio de Educación, Cultura y Deporte de España.

Martín, F. B. (2015). La cultura escolar y el oficio de maestro. Educación XX1, 18(1), 147-166.

Martínez, B. A. (1990). Teoría pedagógica: una Mirada arqueológica a la pedagogía. Pedagogía y Saberes, (1), 7-13.

Messina, R. G. (1999). Investigación en o investigación acerca de la formación docente: un estado del arte en los noventa. Revista Iberoamericana de Educación, (19), 1-54.
Mora, M. Y., Caicedo, N. N. y Mejía, Y. V. (2013). La coherencia y las prácticas pedagógicas (tesis maestría). Universidad de Manizales, Colombia.

Müller de, C. I. (1992). La lucha por la cultura 1. Bogotá, Colombia: Universidad Pedagógica Nacional, CIUP.

Navia, M. A., Navia, M. B. y Timaná, D. E. (2015). Las huellas vitales como generadoras de procesos de transformación de las prácticas pedagógicas (tesis maestría). Universidad de Manizales, Colombia.

Ñaupas, P. H., Mejía, M. E., Novoa, R. E. y Villagómez, P. A. (2014). Metodología de la investigación cuantitativa-cualitativa y redacción de la tesis. Bogotá, Colombia: Ediciones de la U.

Ospina, S. H. y Murcia, P. N. (2012). Regiones investigativas en educación y pedagogía en Colombia: construcción de un mapa de la actividad investigativa de tesis de maestrías y doctorados en el periodo 2000-2010. Manizales, Colombia: Centro de Estudios Avanzados en Niñez y Juventud (Cinde-Universidad de Manizales).

Ossa, M. A. (2015). Lo pedagógico y el maestro investigador. Revista Virtual Universidad Católica del Norte, (44), 102-118.

Palechor, J. E., Mera, M. P. y Zúñiga Q. C. (2016). Prácticas pedagógicas emergentes: retos para una enseñanza situada. Plumilla Educativa, (18), 53-78.

Palomero, P. J., Fernández, D. M. y Teruel, M. M. (2008). La formación de los maestros en España: tiempos de cambio. Revista Educación y Pedagogía, 20(50), 33-58.

Parra, S. R. y Castañeda, B. E. (2014). La vida de los maestros colombianos: interculturalidad $y$ ciudadanía en la escuela. Bogotá, Colombia: Convenio Andrés Bello.

Parra, G. A. y Vallejo, V. C. (2013). Las prácticas pedagógicas en la básica primaria: un espacio para reconocer al otro (tesis maestría). Universidad de Manizales, Colombia.

Parra, S. R., Parra, F. y Carvajal, M. E. (1981). La profesión de maestro y el desarrollo nacional en Colombia. Organización de las Naciones Unidas 
para la Educación, la Ciencia y la Cultura, (UNESCO), Comisión Económica para América Latina y el Caribe (Cepal), Programa de las Naciones Unidas para el Desarrollo (PNUD), Proyecto Desarrollo y Educación en América Latina y el Caribe.

Parra, G. A., Vallejo, V. C. y Loaiza, Z. Y. (2013). Prácticas pedagógicas y reconocimiento del otro: una mirada desde los protagonistas. Revista de Investigaciones, 13(22), 102-115.

Perdomo, M. A., Herrera, C. N., Virguez, R. y Garzón, Z. B. (2016). Prácticas pedagógicas a la luz de las políticas de inclusión educativa (tesis maestría). Universidad de Manizales, Colombia.

Pereira, Á. G., López, C. F. y Ríos, P. A. (2015). Prácticas pedagógicas en cinco lecciones de vida. Plumilla Educativa, (15), 329-346.

Pineda, R. Y. (2007). Contravía Pedagógica. Revista Latinoamericana de Estudios Educativos, 3(1), 77-101.

Pineda, R. Y. y Arbeláez, N. (2009). La práctica pedagógica del maestro de las escuelas normales de Manizales: 1982-1994. Revista Latinoamericana de Estudios Educativos, 5(1), 27-56.

Pineda, R. Y. y Loaiza, Z. Y. (2017). Un análisis del trayecto histórico del currículo en Colombia, segunda mitad del siglo XX. Revista de Investigaciones, 17(29), 150-167.

Pinto, I. T. y García, B. C. (2003). La formación de formadores en Venezuela. La formación de maestros normalistas 1936 -1958 (II). Laurus, 9(16), 129-156.

Pradilla, G. J. (2013). Concepciones de los docentes de la institución técnica la arada sobre la práctica pedagógica y su reconfiguración en el aula de clase (tesis maestría). Universidad de Manizales, Fundación Centro Internacional de Educación y Desarrollo Humano (Cinde), Colombia.

Ramírez, G. A. (2006). Las escuelas normales de Córdoba. Dos instituciones al servicio de la formación de maestros y maestras (1842-1936) (tesis doctorado). Universidad de Córdoba, España.
Rátiva, V. M. (2015). La escritura en los procesos investigativos del ciclo complementario de la Escuela Normal Superior de Cartagena de Indias de 1998 a 2010. Memorias. Pontificia Universidad Javeriana: Colombia. Recuperado de http://wrab2017.com/ javeriana/wp-content/uploads/2015/04/A.pdf

Restrepo, G. B., Villalobos, P. F., Ospina, de G. B. y Buitrago, C. J. (1981). La calidad de la educación en Medellín (tesis posgrado). Universidad de Antioquia, Medellín.

Ríos, B. R. y Cerquera, C. M. (2013). Sobre la formación de maestros en Colombia: una mirada desdela relación entre conocimientos pedagógicos y disciplinares. Pedagogía y Saberes, (39), 21-32.

Ruiz, Q. M., Ortiz, C. C. y Soler, M. J. (2013). Análisis crítico de la práctica pedagógica de docentes en formación. Praxis \& Saber, 4(8), 157-171.

Saker, G. J. (2014). Práctica pedagógica investigativa en las escuelas normales superiores: contexto y pertinencia de la calidad educativa. Educación y Humanismo, 16(26), 83-103.

Saldarriaga, V. O. (2016). La "escuela estallada": diálogos entre dos nociones de práctica pedagógica. Memoria y Sociedad, 20(41), 10-20.

Samboni, I. M., Rengifo, E. H. y Valencia, T. V. (2015). Aproximación a las prácticas pedagógicas en atención de la diversidad en los procesos de lectura y escritura en la I.E. Vasco Núñez De Balboa (Cauca) (tesis maestría). Universidad de Manizales, Colombia.

Sandoval, F. C. (2000). El modelo de formación de maestros en Colombia. La formación de formadores en educación media y básica en los países del convenio Andrés Bello. Memoria del Primer Encuentro de Universidades Pedagógicas, Redes y Facultades de Educación, compilada por Moisés Quito Vidal y Álvaro Campo Cabal. Bogotá, Colombia: Convenio Andrés Bello, Universidad Nacional de Educación, Encuentro de Universidades Pedagógicas, Redes y Facultades de Educación.

Seraphim, P. J. y Lima, J. J. (2018). Las escuelas normales en el Brasil: implementación y consolidación. Helios, 2(1), 45-62. 
Souza, A. J., Gonçalves B. de F. y Pádua, C. L. (2008). As Escolas normais no Brasil: do império à república. Campinas, Brasil: Alínea.

Suárez, O. J. y Urrego, M. L. (2014). Relación familiaescuela: una comprensión al sentido que le dan los docentes desde sus prácticas pedagógicas en la Institución Educativa El Horro de Anserma, Caldas (tesis maestría). Universidad de Manizales y Fundación Centro Internacional de Educación y Desarrollo Humano (Cinde), Colombia.

Taborda, Ch. J. (2009). Sentidos de formación en maestros de una escuela normal superior colombiana (tesis doctoral). Rudecolombia, Manizales, Colombia.

Taborda, Ch. J., Loaiza, Z. Y. y Pineda, R. Y (2012). Una mirada a la experiencia pedagógica en la formación normalista en el contexto del movimiento pedagógico. Revista Latinoamericana de Estudios Educativos, 2(8), 171-209.

Triviño, L. D. (2013). Creencias, práctica pedagógica, saber pedagógico en el habitus del maestro (tesis maestría). Universidad de San Buenaventura, Colombia.

Trujillo, P. N. (2016). Tras la piel del maestro de lenguaje: narrativas de vida y prácticas pedagógicas en la enseñanza de la lectura y la escritura (tesis maestría). Universidad de Antioquia, Colombia.

Valencia, C. C. (2006). Las escuelas normales y la formación del magisterio, primera mitad del siglo $X X$. Manizales, Colombia: Universidad de Caldas, Rudecolombia.
Valencia, C. M. (2013). Prácticas pedagógicas cotidianas de los docentes de la institución Ciudad Puerto Limón (tesis especialización). Universidad Católica de Manizales, Colombia.

Vargas, P. N. (2015). La práctica pedagógica como escenario de formación docente: una mirada al ejercicio docente de los estudiantes de la licenciatura en lingüística y literatura de la Universidad Tecnológica del Chocó (tesis maestría). Universidad de Manizales y Fundación Centro de Estudios Avanzados en Niñez y Juventud (Cinde), Colombia.

Vargas, Z. J. (2016). La autoevaluación institucional y su incidencia en las prácticas pedagógicas. Plumilla Educativa, (17), 272-282.

Vera, N. J. (2011). Reconfiguración de la profesión académica en las escuelas normales. Reencuentro, (62), 82-87.

Zambrano, O. E., Rivera, C. A., Fernández, C. F. y González, S. R. (2014). La práctica pedagógica constructiva: el método de caso. Memorias, 12(22), 81-92.

Zamora, G. L. (2008). Formar educadores para el medio rural o hacer visible lo invisible. Conversaciones Pedagógicas, (6), 95-109.

Zuluaga, G. O. (1999). Pedagogía e historia: la historicidad de la pedagogía, la enseñanza, un objeto de saber. Bogotá, Colombia: Siglo del Hombre Editores, Anthropos. 